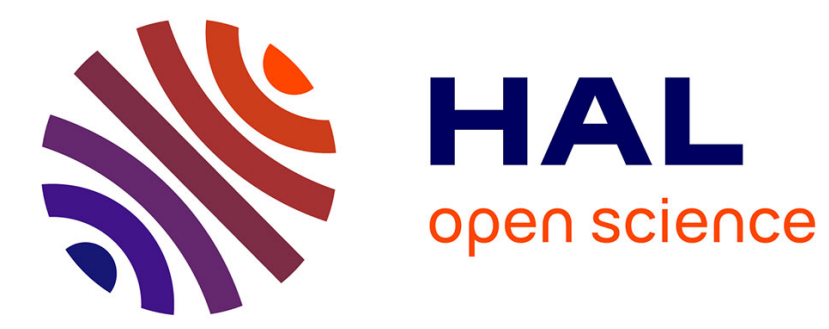

\title{
CHAMBRES PROPORTIONNELLES SOUMISES À DE HAUTS FLUX
}

\author{
J. Thenard
}

\section{To cite this version:}

J. Thenard. CHAMBRES PROPORTIONNELLES SOUMISES À DE HAUTS FLUX. Congres de la Societe Francaise de Physique, 1977, Poitiers, France. pp.C3-17-C3-21, 10.1051/jphyscol:1978304 . jpa-00217408

\section{HAL Id: jpa-00217408 https://hal.science/jpa-00217408}

Submitted on 1 Jan 1978

HAL is a multi-disciplinary open access archive for the deposit and dissemination of scientific research documents, whether they are published or not. The documents may come from teaching and research institutions in France or abroad, or from public or private research centers.
L'archive ouverte pluridisciplinaire HAL, est destinée au dépôt et à la diffusion de documents scientifiques de niveau recherche, publiés ou non, émanant des établissements d'enseignement et de recherche français ou étrangers, des laboratoires publics ou privés. 


\title{
CHAMBRES PROPORTIONNELLES SOUMISES À DE HAUTS FLUX
}

\author{
J. M. THENARD
}

Laboratoire Annecy-le-Vieux Physique des Particules, B:P. 909, 74160 Annecy-le-Vieux, France

\begin{abstract}
Résumé. - Nous présentons les différents problèmes rencontrés lors de l'utilisation de chambres proportionnelles dans des faisceaux de $10^{8}$ particules/seconde. Une revue de différentes mesures expérimentales de l'efficacité est donnée.
\end{abstract}

Abstract. - Results from different groups concerning multiwire proportional chambers in high intensity beam are presented. Different problems to be solved are considered.

1. Introduction. - Depuis le début de l'utilisation de compteurs proportionnels cylindriques, l'effet de haut flux sur l'efficacité des chambres a été noté (Réfs. [1, 2]). Pour les chambres proportionnelles multifils (Réf. [3]), la possibilité de leur utilisation à haute intensité devient un facteur important lorsqu'elles seront implantées dans les faisceaux intenses fournis par le Super-Proton-Synchrotron (SPS) construit au CERN (Genève). Plusieurs faisceaux auront une intensité de $10^{8}$ particules/seconde et une densité de $10^{5}$ particules/seconde $\times$ millimètre carré. Ces détecteurs devront supporter des flux intégrés supérieurs à $3 \times 10^{14}$ particules.

2. Rappels. - Une chambre proportionnelle est constituée de deux cathodes séparées par un plan de fils sensibles.

$d=$ diamètre des fils sensibles.

$S=$ espace cathode-plan de fils.

$s=$ pas de fils sensibles.

$s \ll S$.

Dans le fonctionnement d'une chambre proportionnelle intervient un ensemble de paramètres qui sont liés :

- aux phénomènes intrinsèques dans le gaz:

- ionisation lors du passage de la particule,

- migration des ions et électrons,

- coefficient d'amplification au voisinage du fil,

- à l'électronique associée :

- impédance,

- seuils,

- à la qualité de la construction des chambres :

- mécanique,

- matériau utilisé,

- propreté.

Nous pouvons dire que l'efficacité globale (au sens efficacité de détection et durée de vie) est le produit des efficacités liées à ces paramètres. Nous allons présenter les résultats expérimentaux à haute intensité en tentant de séparer les contributions de chacun de ces points. Cependant, comme nous le verrons en conclusion, il est très difficile d'isoler chacun de ceux-ci.

3. Fonctionnement à haute intensité. 3.1 LIMITATIONS INTRINSÈQUES DUES A L'AMPLIFICATION DANS LE GAZ. - Lors du passage d'une particule, un certain nombre d'électrons primaires ( $\simeq 100 / \mathrm{cm}$ ) sont créés et migrent vers l'anode. A quelques centaines de microns du fil, le champ devient très grand $\left(10^{5} \mathrm{~V} / \mathrm{cm}\right)$. Une multiplication électronique se développe avec un gain de $10^{7}$ (gaz magique). Ces électrons sont captés par l'anode $\left(10^{-10} \mathrm{~s}\right)$. Les ions positifs se déplacent très lentement vers la cathode (quelques 100 microsecondes). Si les électrons primaires créés sur le parcours d'une seconde particule arrivent dans la même zone de développement de l'avalanche, le champ électrique est moins élevé et l'amplification est plus faible : le signal induit pourra ainsi avoir une amplitude trop faible pour déclencher l'électronique.

3.1.1 Calcul macroscopique. - Celui-ci a été fait par Hendricks (Réf. [3]) pour un compteur proportionnel cylindrique. Il a été récemment adapté pour les chambres à dérive (Réf. [4]) et pour les chambres proportionnelles (Réf. [3bis]).

Il s'agit de résoudre l'équation de Poisson tenant compte de la présence, dans l'intervalle cathodeplan de fils, d'une densité de charge positive $\rho$ superposée à la statique créée sur le fil par influence du potentiel $V_{0}$.

Dans le cas d'un fil proportionnel seul, $\rho$ est relié au taux moyen de particules traversant la chambre $R$ par seconde par la formule :

$$
\rho(r)=\frac{n M}{2 \pi \mu} \ln \left(R_{\mathrm{c}} / d\right) \times R
$$


$\mu$ mobilité des ions,

$M$ coefficient d'amplification dans le gaz, $n$ nombre d'électrons primaires créés,

$n \times M \times R$ nombre d'ions créés par unité de temps.

La solution de l'équation de Poisson donne l'expression du champ en deux parties :

$$
E(r)=E_{\text {sans particules }}-E_{\mathrm{c}} \text {. }
$$

Le terme $E_{c}$ peut être assimilé au champ créé par un potentiel $\Delta V$ qui se soustrait au potentiel appliqué au fil, diminuant ainsi l'amplification dans le gaz, donc le gain de la chambre.

Si un effet de charge d'espace macroscopique existe, il doit se manifester par une corrélation des inefficacités entre deux chambres proportionnelles. Les résultats expérimentaux (Fig. 1, Réfs. [5, 6]) tendent à montrer que cette corrélation est faible et peut s'expliquer, comme nous allons le voir par des effets locaux jusqu'aux intensités mesurées.
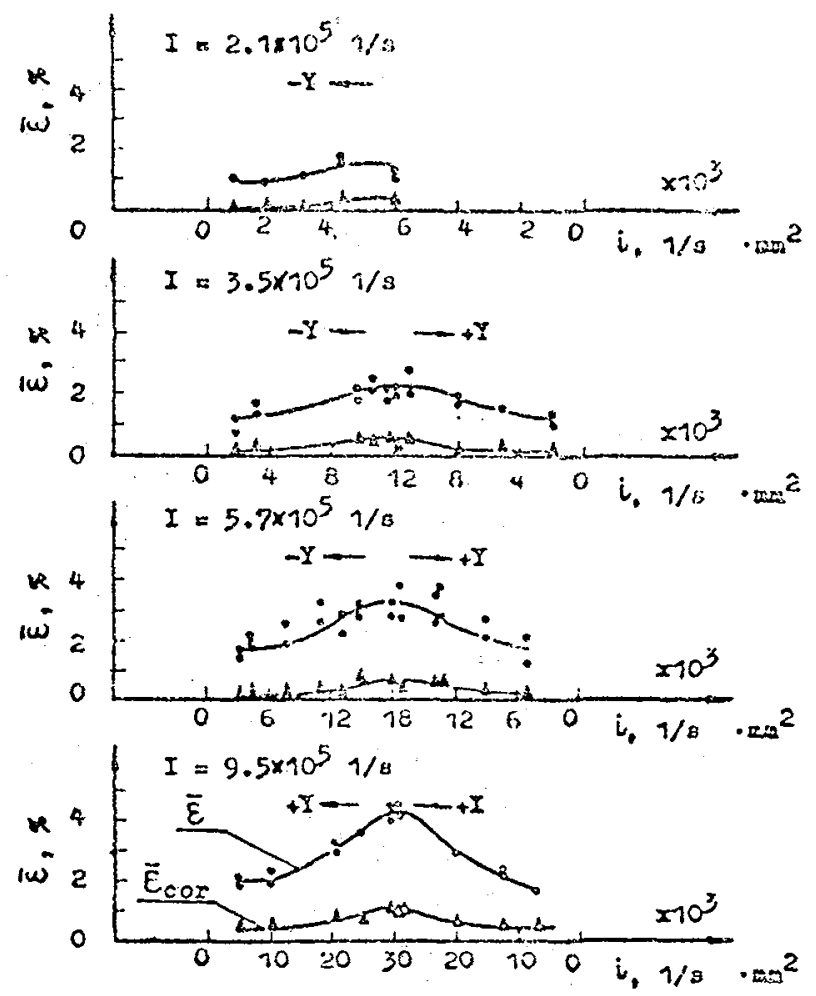

FIG. 1. - Inefficacité $\varepsilon$ et inefficàcité correlée de 2 chambres $\varepsilon$ mesurée le long d'un fil (intensité $i /$ millimètre de fil) pour différents taux de comptage intégré (I) (Réf. [5]).

Il faut noter deux points :

- il est difficile de séparer cette contribution de charge et d'espace de celle due à un temps mort de l'électronique,

- le calcul doit être restreint à une zone au voisinage du fil sensible, où le champ est fort et de symétrie cylindrique. Ceci explique pourquoi l'effet global de charge d'espace n'est pas sensible aux intensités mesurées.
3.1.2 Dimensions de l'avalanche. - Certains ont essayé de mesurer la dimension de l'avalanche (Réf. [7]) dans le gaz magique.

La taille de celle-ci est de l'ordre de $200 \mu$ le long du fil et les ions positifs influencent pendant quelques dizaines de microsecondes cette région du fil.

On peut alors considérer le fil comme insensible à une nouvelle particule arrivant dans cette zone. Avec un flux de $10^{5}$ particules $/ \mathrm{mm}^{2} \times$ seconde, nous avons $6 \%$ de probabilité d'avoir deux particules séparées par $0,2 \mathrm{~mm}$ et $20 \mu \mathrm{s}(20 \%$ avec $0,2 \mathrm{~mm}$ $40 \mu s)$.

L'inefficacité attendue est compatible avec celle mesurée expérimentalement (Réfs. $[5,6]$, Figs. 1, 2), la faible dimension spatiale de l'avalanche pouvant expliquer qu'un faisceau divergent crée une absence de corrélation entre les inefficacités de deux chambres proportionnelles.

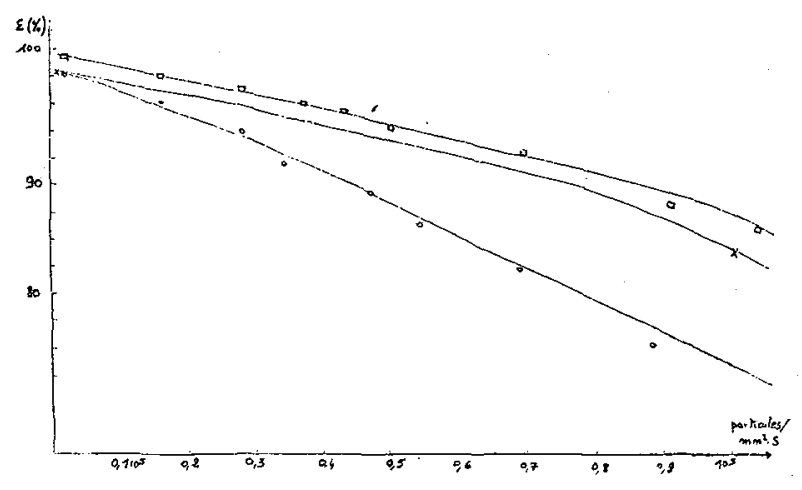

FIG. 2. - Efficacité fonction de l'intensité en particules par millimètre carré par seconde. Trois chambres ont été mesurées (gap $3,2 \mathrm{~mm}, d=10 \mu, \mathrm{pas}=1 \mathrm{~mm}$ ) (Réf. [6]).

\subsubsection{Incidences de ces limitations sur la construc-} tion et le fonctionnement.

- Amplitude du signal :

1'effet d'occultation du champ au voisinage du fil décroît de façon continue après le passage de la première particule ; l'amplitude du signal produit par la chambre au passage d'une seconde particule dépend $\mathrm{du}$ recouvrement spatial et temporel des deux avalanches. L'amplitude moyenne du signal dépend donc de l'intensité de particules traversant la chambre (Figs. 3, 5).

- Haute tension :

le champ étant localement affaibli, on pourrait compenser la charge d'espace en augmentant la haute tension. Cependant, le nombre d'ions créés dans une avalanche est plus grand et la limitation due à la charge devient plus violente, d'où un régime d'équilibre montré par le fait que l'on atteint un plateau d'efficacité à haute intensité (Fig. 4). De plus, accroître la tension de fonctionnement fait décroître la durée de vie de la chambre. 
Gaz :

augmenter la mobilité des ions positifs permettrait de diminuer le temps pendant lequel la seconde particule est sensible au passage de la première. Deux mélanges gazeux ont été testés à haute intensité :

$$
\begin{array}{lll}
\mathrm{Ar}+\mathrm{CO}_{2} & \text { mobilité } & \mu=1,09 \times 10^{-4} \mathrm{~m}^{2} \mathrm{~V}^{-1} \mathrm{~s}^{-1} \\
\mathrm{Ar}+\text { isobutane + méthylal } & \mu=0,89 \times 10^{-4}
\end{array}
$$

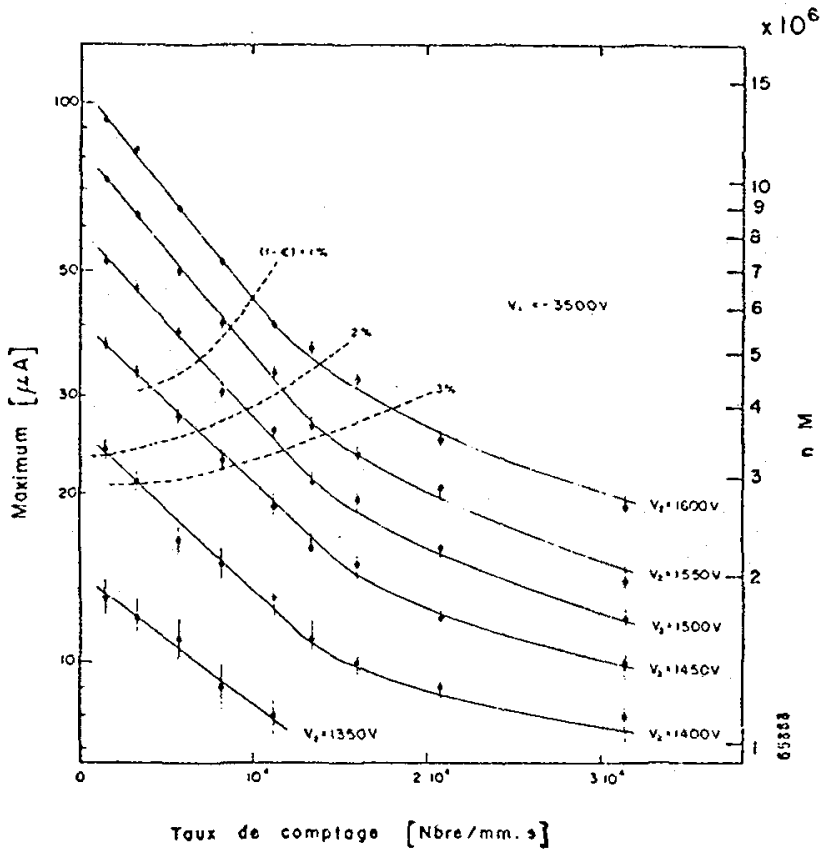

FIG. 3. - Maximum du spectre d'amplitude des signaux de chambre proportionnelle en fonction du taux de comptage (particules au minimum d'ionisation (Réf. [4]).

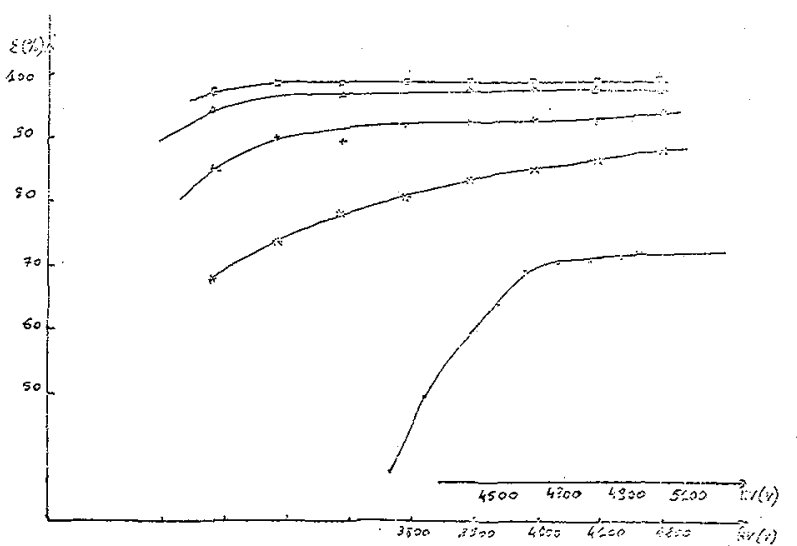

Fig. 4. - Efficacité pour différentes tensions à différentes intensités particules au minimum d'ionisation (Réf. [6]).

$* 1 \times 10^{5}$ particules $/ \mathrm{mm}^{2} \times \mathrm{s}$

$+0,5 \times 10^{5}$

$\triangle 0,16 \times 10^{5} \quad$ seuil $2 \mathrm{~A}$

$\square 0,02 \times 10^{5} \quad$ gaz magique

source (Réf. [8])

- $10^{5} \mathrm{p} / \mathrm{mm}^{2} \times \mathrm{s}$.

Les différents résultats n'ont pas été normalisés à $100 \%$ à basse intensité.

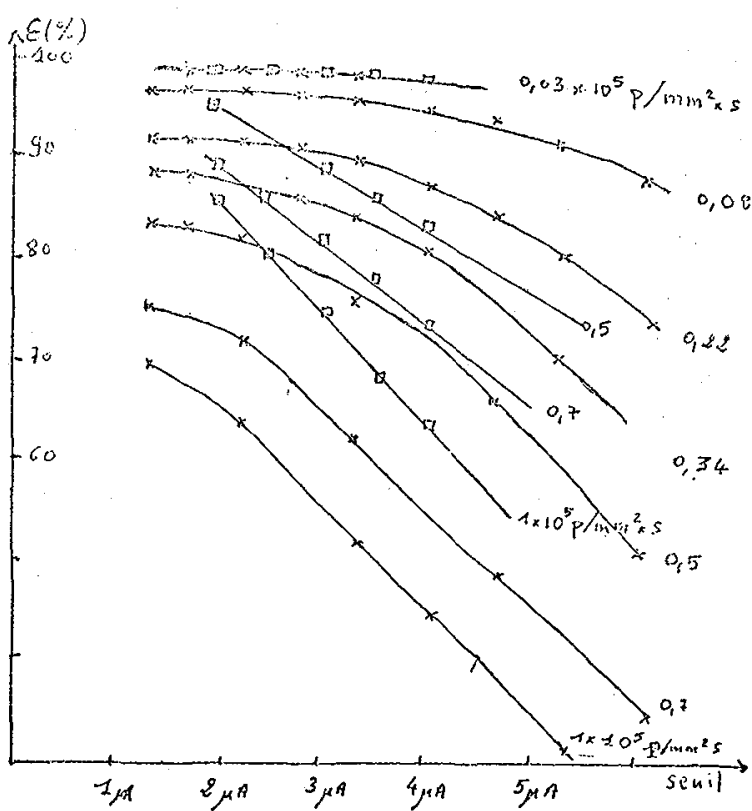

FIG. 5. - Efficacité pour différentes intensités fonction du seuil de l'amplificateur (Réfs. $[6,8]$ ).

Il y a peu de différence. Pour notre part, nous avons choisi d'utiliser autant que possible le mélange magique pour les raisons données par Charpak (Réf. [7]). L'utilisation de l'hélium augmenterait la mobilité, mais réduirait l'amplitude des signaux.

- Espacement des fils :

les résultats précités concernent tous des chambres au pas de $1 \mathrm{~mm}$, le diamètre étant de 10 microns. Utiliser des chambres au pas de $0,5 \mathrm{~mm}$ réduirait les inefficacités d'un facteur 2 ; cependant le diamètre du fil devrait alors être de l'ordre de 5 microns (le rapport $s / d$ devant être à peu près conservé). D'autre part, la longueur effective de multiplication devient plus petite donc le gain diminue.

En fait, seuls des prototypes ont fonctionné jusqu'à présent avec des fils de diamètre $5 \mu$. Les problèmes de mécanique avec des fils de cette taille deviennent cruciaux.

3.2 Limitations dues a L'Électronique. - A haut flux, il est difficile de considérer le fonctionnement de la chambre indépendamment de l'électronique : 
- l'amplitude des signaux produits par la chambre décrô̂t avec l'intensité (Fig. 5),

- le taux instantané par fil devient très grand (10 $\mathrm{MHz}$ ).

Il faut donc un amplificateur avec un temps d'occupation faible (ceci exclut les retards par monostable unique), une impédance d'entrée basse pour éviter l'empilement (à haut taux de répétition le seuil d'un ampli avec couplage capacitif dérive), un seuil très faible (inférieur à 2 microampères).

Il faut noter qu'il est difficile de normaliser les résultats provenant de mesure avec différents amplificateurs : ceux-ci peuvent être sensibles à la forme du signal, aux capacités parasites à ce niveau de courant d'entrée.

Un paramètre important est la dépendance du seuil en fonction du temps entre 2 signaux arrivant sur l'amplificateur (Fig. 5bis). Dans ce cas précis (Réf. [6]), il n'y a pas $100 \%$ de temps mort mais une variation du seuil effectif donc un temps mort fonction de l'amplitude des signaux et de leur séparation en temps. Ceci montre qu'il est très difficile d'isoler des inefficacités réellement liées aux phénomènes intrinsèques dans la chambre et celles liées à l'électronique.

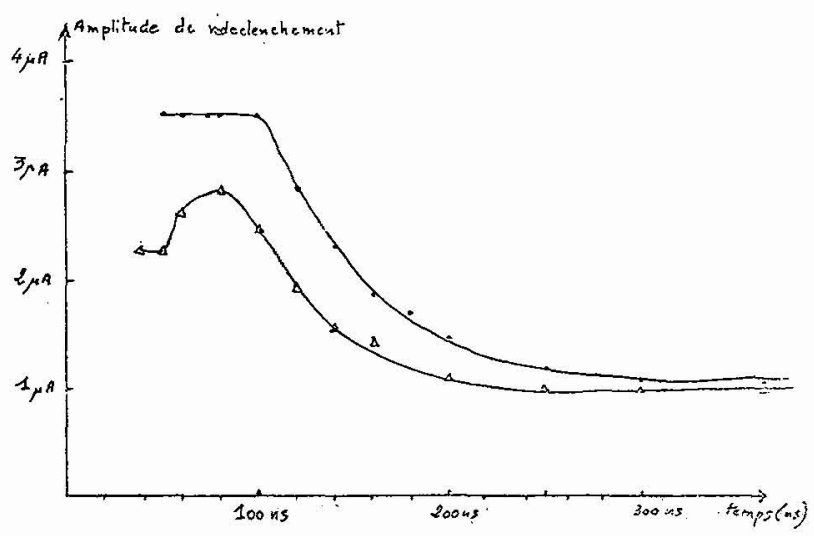

FIG. 5 bis. - Seuil effectif de l'amplificateur fonction du temps de séparation entre signaux venant des chambres.

- 10 fois au-dessus du seuil

$\times 20$ fois au-dessus du seuil (Réf. [6]).

\subsection{DOMMAGES LIÉS AUX HAUTES INTENSITÉS. -} Nous pouvons les diviser en 2 aspects :

3.3.1 Dommages causés par la dose instantanée. - Si le fil présente des dépôts ou des accidents, à haute intensité une chimie locale prend naissance dans la zone fortement ionisée créée par l'imperfection du fil; cette chimie est le départ pour un passage privilégié d'un courant de fuite empêchant le fonctionnement de la chambre. L'homogénéité de la tension des fils ainsi que la planéité des cathodes sont des paramètres importants. Les produits utilisés (décapant, soudure sans collophane) pour souder les fils et les nettoyer doivent être soigneusement choisis. En fait il existe un niveau nécessaire de propreté et une qualité de construction tels qu'à une intensité de particules donnée il n'y ait pas d'accrochage de la chambre.

Nous avons testé les chambres sous azote (chambre remplie d'azote). Les photographies de ce test ne doivent révéler aucun point ou fil brillant (Fig. 6). Le plateau d'efficacité mesuré à basse intensité doit avoir une largeur supérieure à $1000 \mathrm{~V}$ pour qu'une chambre puisse fonctionner à haute intensité sans destruction en quelques minutes.

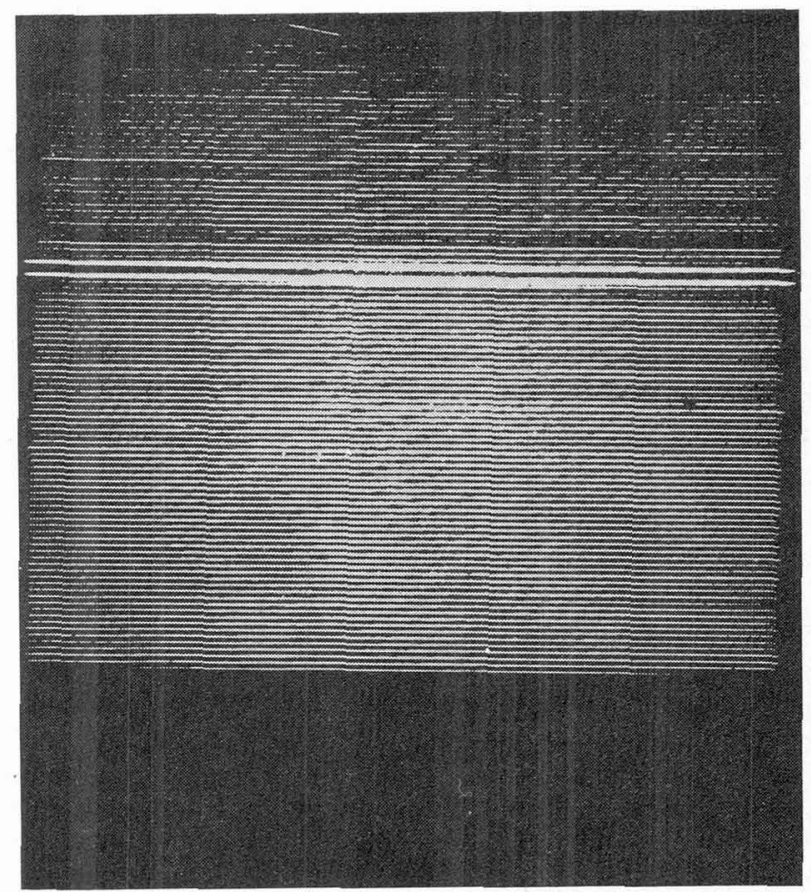

FIG. 6. - Test à l'azote (Réf. [6]). La chambre remplie d'azote est mise sous tension $(5000 \mathrm{~V})$. La photographie montre les défauts de cette chambre

- fils détendus

- points lumineux (fils abîmés)

- zone morte indiquant un défaut de planéité.

3.3.2 Dommages causés par la dose intégrée. Ces dommages qui se manifestent par un courant de fuite qui croît et un point de fonctionnement qui se déplace vers les hautes tensions sont dus à une chimie dans le gaz avec formation de produits secondaires qui migrent vers la cathode ou se déposent sur les fils sensibles. Sur la cathode ces produits donnent naissance aux mêmes phénomènes que ceux décrits précédemment. Sur le plan de fil ces dépôts rendent les fils inefficaces.

En particulier, dans le gaz magique, le méthylal peut contenir des péroxydes; il peut s'en former d'autre part à partir du fréon malgré son potentiel d'ionisation élevé.

Ces peroxydes attaquent le cuivre et ces dépôts forment des arborescences du type figure 7. 


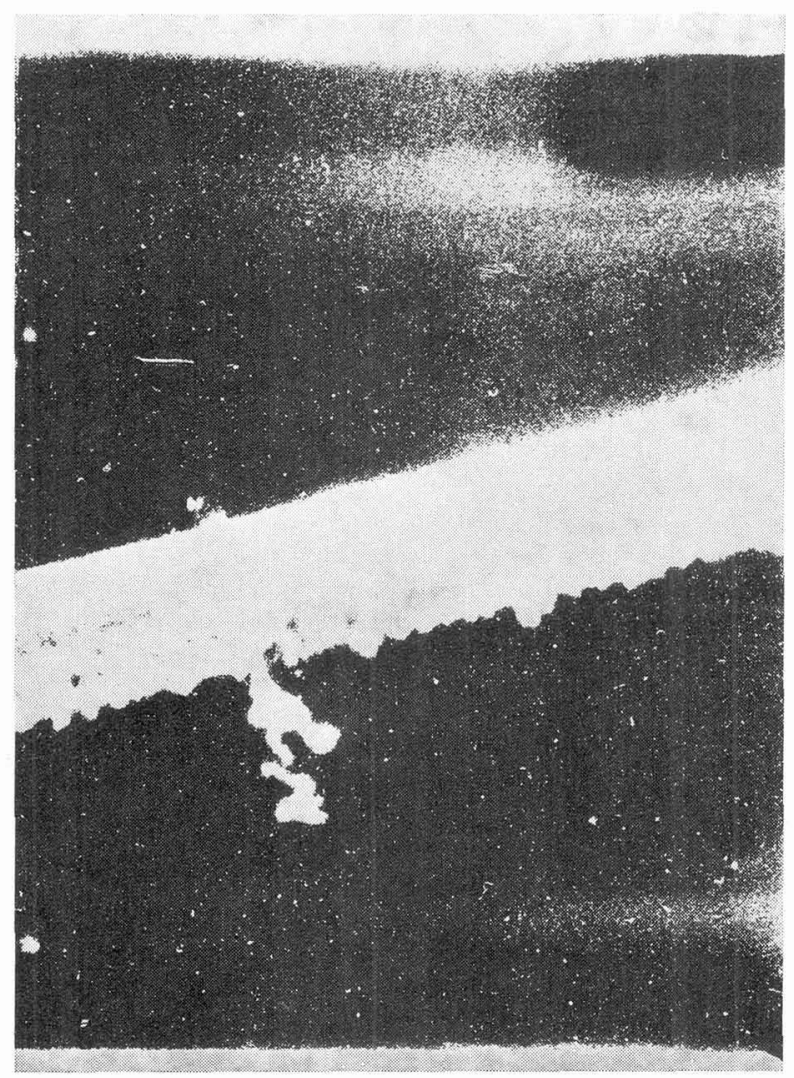

FIG. 7. - Dépôt sur un fil de garde $(\mathrm{Cu}-\mathrm{Be}, 100 \mu)$ après irradiation (courant dans la chambre $800 \mu \mathrm{A}$, source $80 \mathrm{mCi}$ ).

Le choix du type de cathode est important. Avec les cathodes en aluminium il $y$ a la formation d'alumine isolante d'où destruction.

Les cathodes en carbone déposé sur mylar ont été testées et sont utilisées ; le choix du carbone déposé est important pour la durée de vie $\left(^{1}\right)$.

Les grilles en acier inoxydable sont difficiles à nettoyer mais l'avantage est qu'elles laissent migrer le condensat formé dans la chambre vers les parois extérieures de la chambre.

(1) Le Du, Palanque ; Communication privée.

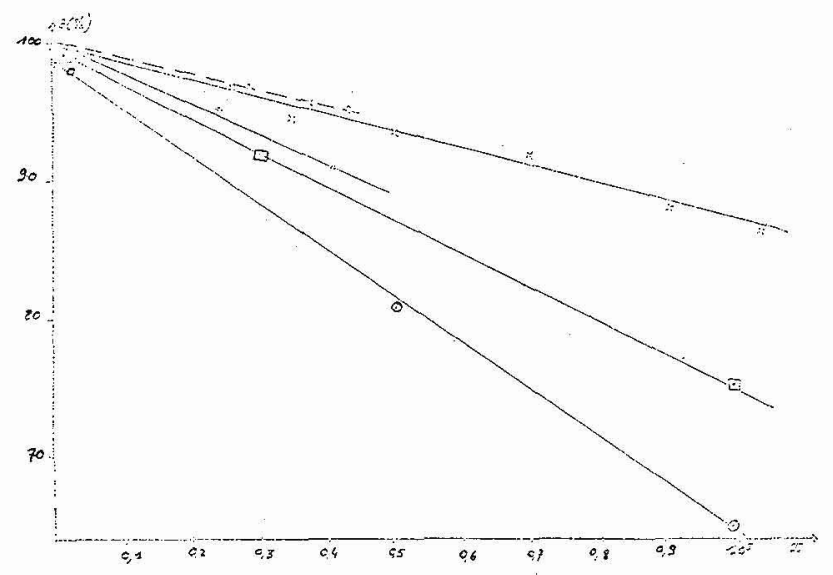

FIG. 8. - Efficacité fonction de l'intensité (Réfs. [5, 6, 7, 8, 11])

$\triangle$ réf. [4]

$\times$ réf. $[6]$

- réf. [5]

$\odot$ réf. [8]

๑ réf. [11]

- efficacité corrélée de 2 chambres (Réfs. $[5,6]$ ).

Tous les résultats donnés concernent des chambres avec des fils en tungstène doré de 10 microns.

4. Conclusion. - Les différentes mesures expérimentales d'efficacité qui ont été faites sont portées sur la figure 8. Les différences entre ces résultats sont explicables par des différences de gaz, d'électronique, de conditions de test (source ou particules au minimum d'ionisation). Comme nous l'avons vu, ces résultats intègrent les chambres et l'électronique associée et il est difficile de séparer les paramètres. Toutes semblent converger vers des inefficacités de l'ordre de $15 \%$ à $10^{5}$ particules au millimètre carré seconde. De plus la mesure de corrélation faite avec plusieurs chambres à Léningrad (Réf. [5]) et au CERN (Réf. [6]) montre la possibilité d'abaisser cette inefficacité en doublant les plans de fil.

A $10^{5} \mathrm{p} / \mathrm{mm}^{2} \times \mathrm{s}$ l'efficacité obtenue en utilisant 2 chambres est de $96 \%$ montrant ainsi la possibilité d'utilisation dans le faisceau du SPS. Le problème principal reste la durée de vie de ces chambres à haute intensité.

\section{Bibliographie}

[1] WILKINson, Ionization Chambers (Cambridge University Press, London) 1950.

[2] SpIELberg, Rev. Sci. Instrum. 37 (1968) 1268.

[2bis] CHARPACK, Nucl. Instrum. Methods 99 (1972) 279-284.

[3] HENDRICKS, Rev. Sci. Instrum. 40 (1969) 1216.

[3 bis] MAKOWSKI et al., CERN/DPHII/73-3.

[4] Schultz, Thèse Université Louis-Pasteur, Strasbourg.
[5] DAMASKINSKY et al., Leningrad Nuclear Institute/305.

[6] AUBERT, J. J. et al., to be published.

[7] BRESKIN et al., Nucl. Instrum. Methods 124 (1975) 189-214.

[8] LE DU et al., CERN OM/SPS/75-46.

[9] CHARPACK et al., Revue Phys. Appl. 12 (1977) 67.

[10] ALANDRY, Service Toxicologie Industrielle (Genève).

[11] DUERDOTH et al., Nucl. Instrum. Methods 129 (1975) 461-465. 